\title{
Feasibility and Acceptability of an Internet-Based Program to Promote Physical Activity in Adults With Cystic Fibrosis
}

\author{
Narelle S Cox PT PhD, Jennifer A Alison PT PhD, Brenda M Button PT PhD, \\ John W Wilson MD PhD, and Anne E Holland PT PhD
}

\begin{abstract}
BACKGROUND: Lifelong physical activity is an important component of the therapeutic management of patients with cystic fibrosis (CF). Use of the internet to monitor and encourage participation in physical activity has not been assessed in adults with CF. We aimed to establish the feasibility and acceptability of a specifically developed internet-based program to monitor and encourage physical activity participation in adults with CF. METHODS: Subjects were recruited at hospital discharge to trial an internet-based physical activity program (ActivOnline) for 8 weeks, which incorporated fortnightly telephone consultation to support physical activity behavior change. Acceptability of the program was assessed by semistructured interview, as well as subject-rated system usability and perceived benefit using Likert scales. Feasibility was assessed by frequency of access of the online site and number of physical activity sessions recorded. RESULTS: Ten subjects were recruited who rated system usability and perceived benefit favorably (median score usability of $89 \%$ [interquartile range of 84-95\%]; median score of perceived benefit (maximum of 5) of 4 [interquartile range of 3-4.8]). During interviews, subjects described a positive reaction to receiving graphical representation of their activity participation; however, $80 \%$ would have preferred a more mobile interface such as an app. Subjects accessed ActivOnline on a mean \pm SD of $13 \pm 11$ occasions over 8 weeks and recorded a mean of 35 (range of 15-57) physical activity sessions. CONCLUSIONS: Use of an internet-based program to encourage participation in physical activity was both feasible and acceptable to adults with CF. Feasibility may be further improved with the ability to access the program through a mobile application. Key words: cystic fibrosis; physical activity; internet; activity promotion; telehealth. [Respir Care 2015;60(3):422-429. (C) 2015 Daedalus Enterprises]
\end{abstract}

\section{Introduction}

Regular participation in moderate-to-vigorous physical activity by healthy adults has numerous health benefits, including improved cardiorespiratory fitness and bone density and reduced risk of non-communicable disease and

Drs Cox and Holland are affiliated with the Department of Physiotherapy, La Trobe University, Alfred Health Clinical School, Melbourne, Victoria, Australia. Dr Holland is also affiliated with the Department of Physiotherapy, Alfred Health, Melbourne, Victoria, Australia. Dr Alison is affiliated with the Department of Respiratory Medicine, Royal Prince Alfred Hospital, and the Discipline of Physiotherapy, Faculty of Health Sciences, The University of Sydney, Sydney, New South Wales, Australia. Drs Button and Wilson are affiliated with the Department of AIRmed, Alfred Health, Melbourne, Australia, and the Department of Medicine, Alfred Campus, Monash University, Melbourne, Victoria, Australia. depression. ${ }^{1}$ Despite the benefits of participation in regular physical activity, only $25-30 \%$ of the healthy population over $15 \mathrm{y}$ of age reported participating in repeated sessions of at least $30 \mathrm{~min}$ of moderate activity per week. ${ }^{2,3} \mathrm{In}$ cystic fibrosis $(\mathrm{CF})$, routine physical activity participation may improve exercise tolerance and relieve breathless-

\footnotetext{
Dr Cox was supported by a National Health and Medical Research Council PhD scholarship and a Cystic Fibrosis Australia PhD stipend. The other authors have disclosed no conflicts of interest.

Correspondence: Narelle S Cox PT PhD, Department of Physiotherapy, Alfred Health Clinical School, La Trobe University, Level 4, The Alfred Centre, 99 Commercial Road, Melbourne, Victoria 3004, Australia. E-mail: n.cox@latrobe.edu.au.
}

DOI: $10.4187 /$ respcare. 03165 
ness, as well as positively influence bone accretion, blood glucose control, and clearance of pulmonary secretions. ${ }^{4}$ Such health benefits may result in improvements in exercise capacity, respiratory function, and quality of life, all of which have been associated with improved prognostic outcomes..$^{5-7}$ However, in a survey of adults with $\mathrm{CF}$, $<20 \%$ reported performing exercise sufficient to cause shortness of breath or sweating on $>4$ occasions per week over the previous 6-month period. ${ }^{8}$

In other respiratory disorders such as COPD, there is an inverse association between the amount and intensity of physical activity undertaken and the need for hospitalization, ${ }^{9}$ health-care utilization, ${ }^{10}$ and mortality. ${ }^{11}$ Strategies to effect behavior change and increase physical activity participation in patients with COPD include supervised exercise training, such as in pulmonary rehabilitation, or educational or motivational sessions. ${ }^{12}$ However, for patients with $\mathrm{CF}$, group-based exercise programs are precluded due to the need for patient segregation and isolation from their peers to prevent cross-infection. ${ }^{13}$ Other activitypromotion strategies investigated in respiratory populations have used telehealth technologies such as walking programs with pedometer feedback and internet-based monitoring ${ }^{14}$ and text-messaging support. ${ }^{15}$

Patients with CF have described themselves as confident in the use of health-care technology; however, telehealth applications used by this group have been limited mostly to transfer of symptom data and physiologic monitoring. ${ }^{16}$ Reports of telehealth-based exercise interventions for patients with $\mathrm{CF}$ are lacking. ${ }^{17}$ The choice of the internet as the means to deliver a physical activity program allows participants the convenience of accessing the program at a time and place of their choosing ${ }^{18,19}$ and does not place a geographic limitation on potential participants. ${ }^{20}$ Consequently, internet-based programs may help overcome barriers to exercise reported by participants attending hospital-based exercise programs (eg, transport and time $)^{21}$ and may aid in improving exercise program adherence. ${ }^{18}$ Evidence from patient preference studies and successful physical activity-promotion strategies suggests that effective strategies include the exclusive targeting of physical activity, ${ }^{12}$ use of behavioral strategies such as feedback and goal setting, ${ }^{12,18,22}$ and capacity for selfmonitoring. ${ }^{12}$ Additionally, reports of patient preferences relating to internet-based physical activity programs included easy program accessibility with simple interactive features; the ability to document, track, and monitor personal progress; and the capability to access expert advice via e-mail. ${ }^{23}$

The aim of this study was to assess the feasibility and acceptability of an internet-based program specifically designed to encourage participation in physical activity by adults with CF.

\section{QUICK LOOK}

\section{Current knowledge}

Pulmonary rehabilitation including an exercise component has been shown to improve exercise tolerance, reduce dyspnea, and enhance secretion clearance in patients with cystic fibrosis. Methods to encourage compliance and monitor exercise in these patients requires a multimodal approach.

\section{What this paper contributes to our knowledge}

In a small group of 10 subjects, use of an internet-based program to encourage physical activity participation was feasible and acceptable to adults with cystic fibrosis. Subjects suggested that the program could be further improved by enabling activity monitoring to be accessed via a mobile application.

\section{Methods}

Adults with a confirmed diagnosis of $\mathrm{CF}$ were invited to participate in the study at the time of hospital discharge after treatment of a respiratory exacerbation with intravenous antibiotics. Potential subjects were excluded if they had a comorbidity that limited mobilization or physical activity participation, had received previous lung transplantation, were pregnant, or did not have access to the internet at home. A researcher not involved in their clinical care recruited subjects, with study participation commencing when subjects were discharged from in-patient services. As this was a feasibility and acceptability trial of internet technology, a power calculation was not performed. This study was approved by the ethics committees of The Alfred Hospital and La Trobe University in Melbourne, Australia. All subjects provided written informed consent. Baseline assessment was completed at hospital discharge, followed by 1 week of habitual physical activity monitoring using the SenseWear Pro3 armband (BodyMedia, Pittsburgh, Pennsylvania). Use of the internet-based physical activity program commenced 1 week following hospital discharge (Fig. 1).

\section{Internet-based Physical Activity Program}

The internet-based physical activity program ActivOnline (www.activonline.com.au, accessed October 21, 2014) was specifically designed by us for this study. The program provided users with real-time graphical representation of their physical activity entries (in particular, activity duration and daily step count), as well as a written display of previous entries (Fig. 2). There was an inbuilt messag- 
WEEK
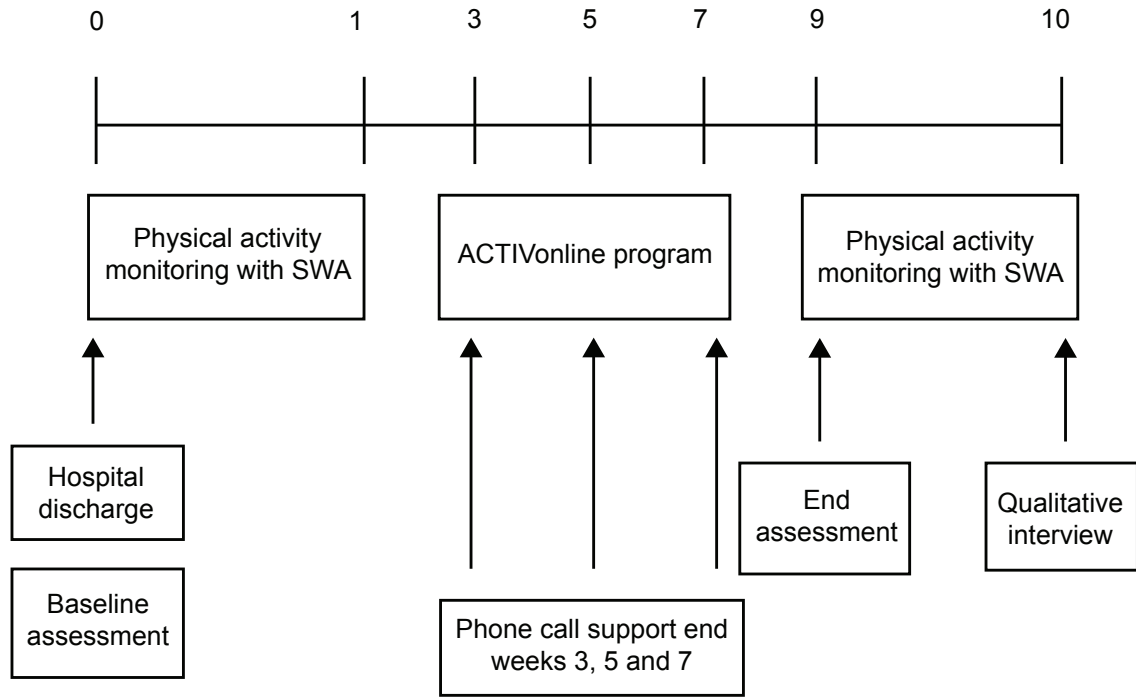

Fig. 1. Timeline of assessments and intervention. SWA $=$ SenseWear Pro3 Armband.

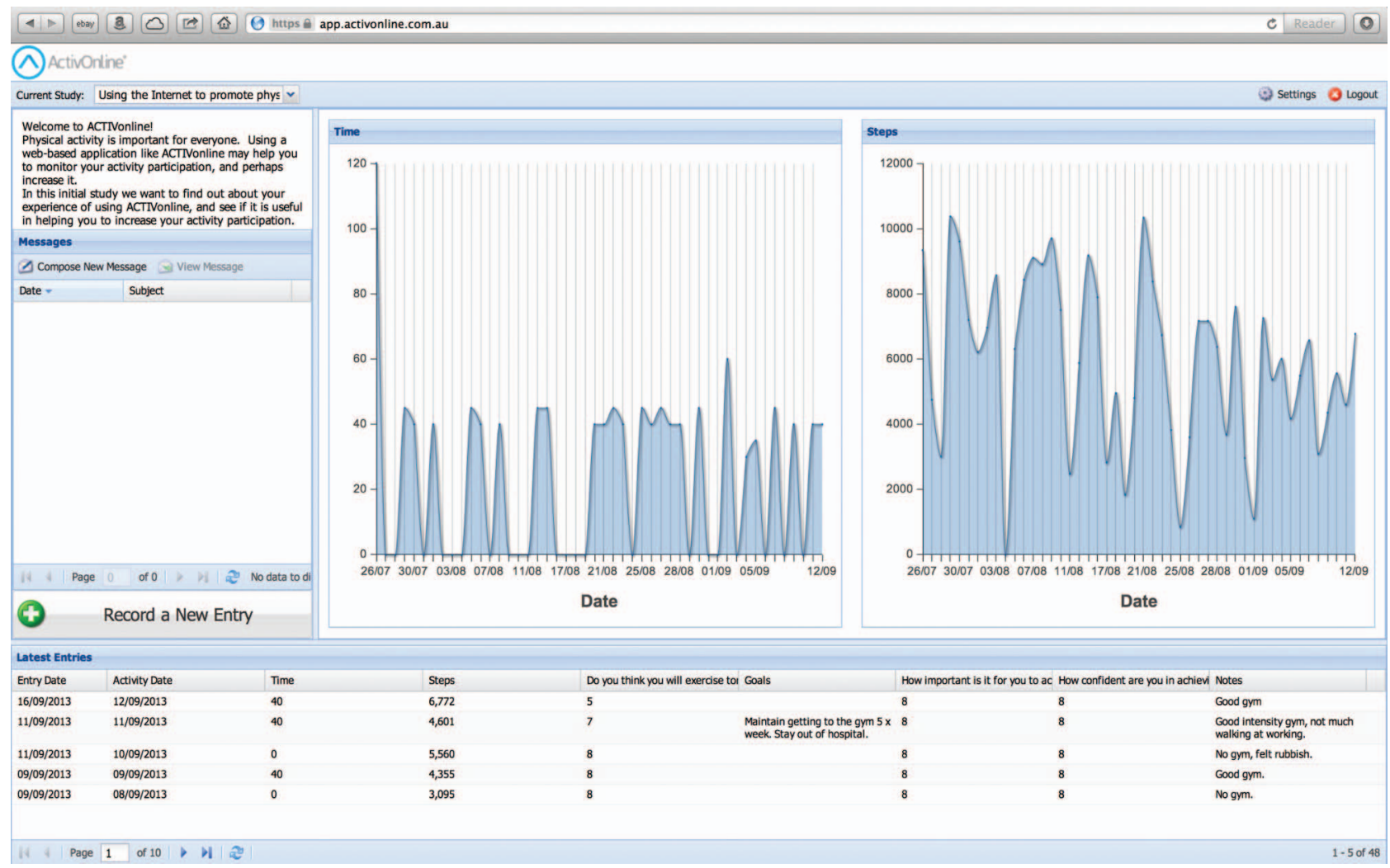

Fig. 2. Example screen shot of subject's personal ActivOnline web page.

ing system for direct communication between subjects and the research team, and subjects received an automated e-mail message reminding them to log into the program when $>3 \mathrm{~d}$ had elapsed since recording the previous entry. Each subject was provided with an individualized user- name and password and instructed how to enter data relating to daily step count, daily time spent in physical activity, and physical activity goals. All subjects received a telephone consultation before using ActivOnline and each fortnight from a physiotherapist experienced in the man- 


\section{Internet-Based Program to Promote Physical Activity in CF}

agement of CF. The aim of the telephone consultation was to foster goal setting by discussing barriers and facilitators to physical activity and building motivation for physical activity participation. Each subject was provided with a pedometer (Omron HJ-112, Omron Healthcare, Bannockburn, Illinois) to monitor daily step count during the program. To ensure the security of information entered into the program, and to maintain subject privacy, security measures included access protection to the internet site by unique username and password and use of 128-bit SSL security to provide encrypted data transfer at all times.

\section{Outcome Measures}

Feasibility of ActivOnline as a program to encourage physical activity participation by adults with CF was assessed by the frequency of site access, number of activity sessions recorded, and number of telephone consultations undertaken. ActivOnline acceptability was assessed through semistructured interviews ${ }^{24}$ and subject preference ratings using Likert scales. Semistructured interviews with an independent researcher were recorded for transcription of responses. Interview questions explored subjects' attitudes toward physical activity, competence with technology, positive and negative functions of the internet site, effect of the program on behavior change relating to physical activity, and preferred methods for improving physical activity participation. Subjects also rated perceived benefit of web-based exercise rehabilitation and their interest in continued use of the ActivOnline program using a 5-point Likert scale. User experience of the ActivOnline system was assessed with the 10-question System Usability Scale (SUS). ${ }^{25}$ The SUS is a free tool with good internal consistency designed to assess the user's experience of technology. ${ }^{25}$ The questionnaire consists of 10 questions regarding aspects of a system's use, such as the need for support, training, and complexity, each of which is scored on a 5-point Likert scale (strongly agree to strongly disagree). The SUS has been found to be superior to other similar tools in assessing web site usability ${ }^{26}$ and has a maximum high score of 100 points.

\section{Secondary Outcome Measures}

Secondary outcome measures of physical activity participation, health-related quality of life, and exercise capacity were assessed before and at the completion of the 8-week trial of ActivOnline. Quality of life was assessed using the revised edition of the Cystic Fibrosis Questionnaire (CFQ-R). ${ }^{27}$ Higher scores indicate better quality of life. The Modified Shuttle Test, a commonly used field test of aerobic capacity that is a reliable and valid measure of exercise capacity in adults with $\mathrm{CF},{ }^{28}$ was used to assess exercise capacity.
Participation in physical activity was monitored for 1 week immediately after hospital discharge and before trialing the ActivOnline program using the SenseWear Pro3 armband. The SenseWear Pro3 armband is a portable multisensor armband that contains a bi-axial accelerometer and sensors that measure galvanic skin response, heat flux, and skin temperature. Data are stored minute by minute, and a proprietary algorithm (version 6.1) estimates energy expenditure. Step count is also recorded. The SenseWear Pro3 armband is worn on the right upper arm and activates automatically on contact with the skin; it is removed only for bathing or water activities. It was determined a priori that a minimum of $3 \mathrm{~d}$ of wear ${ }^{29}$ of at least $10 \mathrm{~h}$ on each day was required for data to be included in the analysis. ${ }^{6}$

Analyses were performed using statistics software (PASW Statistics 20, SPSS, Chicago, Illinois). Paired values were compared using Wilcoxon signed-rank tests, and $P<.05$ was considered significant. Results are expressed as mean $\pm \mathrm{SD}$ or median (interquartile range) unless stated otherwise. De-identified interview transcripts were independently reviewed by 2 researchers, and line-by-line analysis was conducted to develop codes and identify themes arising in the interviews.

\section{Results}

Ten adults with CF (4 males) were recruited, and there were no dropouts during the study period. The mean \pm SD age of subjects was $30 \pm 8 \mathrm{y}$, and the mean $\mathrm{FEV}_{1}$ on hospital discharge was $50 \%$ of predicted (range of 29 $88 \%$ of predicted). All subjects were pancreatic insufficient, and 2 subjects had CF-related diabetes.

\section{Feasibility}

Each subject logged into ActivOnline a mean of $13 \pm 11$ times over the 8-week intervention period. Subjects recorded a mean of 35 (range of 15-57) physical activity sessions during the intervention period, equating to a mean of 4 recorded sessions of physical activity each week. Seven subjects completed all 4 telephone consultations during the study period; the remaining subjects undertook one $(n=1)$ or $2(n=2)$ of the scheduled telephone consultations to foster goal setting.

\section{Acceptability}

Nine qualitative interviews were conducted by telephone, with one undertaken in person. Responses were grouped relating to internet familiarity and use, timeliness of ActivOnline intervention, technology functionality, and 


\section{Internet-Based Program to Promote Physical Activity in CF}

usefulness for goal setting and performance management. Subjects were all familiar with using web-based technology and reported using multiple devices to access the internet on at least a daily basis:

[I access the internet] ... multiple times a day ... I pretty much live on the internet to be honest. (Subject 9)

Nine subjects accessed the internet predominantly via a mobile device:

... mostly my mobile telephone, then second by the iPad, and third by the computer. But the iPad and the computer are a bit more of a hassle because they're usually at home, where I'm not. So usually the mobile telephone. (Subject 7)

None of the subjects accessed ActivOnline on a daily basis. Although subjects reported that daily access would be feasible, allowing time for data entry on a couple of occasions per week was a more realistic expectation:

You probably need to do it every 3 days. Any more than that, you're starting to forget what you've done, unless you write it down. (Subject 3)

A major hurdle to data entry for 4 subjects was remembering to undertake the task. These subjects felt they may have benefited from a data entry reminder:

It's really easy to log on and get it all done; it's just a case of remembering to do it, that's all. (Subject 10)

One subject reported that the inbuilt reminder e-mail system was useful for overcoming this issue:

Also, it would send me nice e-mails to remind me that I hadn't put entries for like 3 days. So then if I like forgot to put something, I'd be like, oh yeah, now I remember, so that was really handy. (Subject 5)

Use of ActivOnline immediately upon hospital discharge was viewed by subjects as being potentially helpful in aiding return to an exercise routine and monitoring exercise performance following a respiratory exacerbation:

I think it's a good short-term program. I don't think it's something I could do long term ... it was good after an admission to get back into exercise and monitor what I was doing. (Subject 3)

[Using following discharge] ... it could be (good) because I'd like to see how quickly I recover. (Subject 5)
Given their preference for accessing the internet via mobile devices, subjects described the functionality of the current software version as being less robust on mobile devices compared with a computer:

Some of the graphs and things didn't show up that well; they certainly showed up better on the laptop. (Subject 1)

It was very jumpy using it on the iPad. It didn't like you putting in the data. It would kick you out of the fields quickly, so the stability of using it on the iPad was a bit of a trick. (Subject 6)

Eight subjects indicated that being able to enter activity data at the time and location of physical activity participation would be ideal and could improve frequency of data entry:

[If you had an app] ... you just punch it in and go. (Subject 3)

[Ability to access via telephone]... definitely ... because then you could just do it on the go. (Subject 8)

Subjects indicated a desire for recording more detailed information possibly including integration with other physiologic monitors, a better distinction between dedicated exercise and incidental activity, and the use of Global Positioning System (GPS) technology to track distance and step count, thus negating the need to wear a pedometer:

... you can have a GPS for running and distance, so you can get a lot more data depending on what mode of exercise you're in. (Subject 6)

I thought it would have been even more interesting if it had like converted your number of steps into how far you walked. (Subject 8)

It did get a little annoying sometimes, depending on what outfits you were wearing or where you were going to carry a pedometer somewhere. So there were a couple of times I wrote in the notes section 'pedometer would not work with my dress.' (Subject 4)

I thought maybe the definition of what you should put in there and what you should leave out needs to be a bit clearer because if, say you do a lot of incidental type exercise, ... . it's really hard to measure that type of thing when you might have just been walking around your house or shopping. (Subject 4) 
Table 1. Preferred Timing of Use of ActivOnline

\begin{tabular}{lc}
\hline \hline \multicolumn{1}{c}{ Timing } & $\begin{array}{c}\text { Subjects, } \\
n(\%)\end{array}$ \\
\hline $\begin{array}{l}\text { During home-based intravenous } \\
\text { antibiotic treatment }\end{array}$ & $4(40)$ \\
Following hospital discharge & $3(30)$ \\
All the time & $2(20)$ \\
Twice/y & $1(10)$ \\
Never & $0(0)$ \\
\hline
\end{tabular}

Seven subjects specifically described the graphical representation of their activity as a positive means to monitor and track their activity performance:

I liked being able to track the graphs ... being able to have the visual of the overview of the week because it helped you try to aim for some consistency rather than being up and down. (Subject 7)

I found it useful to be able to see the graphs of the activity; I could see in my own mind a correlation between how much activity I was doing and how I was feeling. (Subject 10)

More than half of subjects reported using ActivOnline as a prompt to maintain or change their exercise behavior and to help them set goals for activity participation:

\begin{abstract}
It was kind of like a bit of an external check that makes your brain think 'I may not want to go to the gym, but I'll do it anyway because I've got to put it into here' ... the whole thing that you're writing things down and stuff is sort of motivation in itself to sort of remain consistent in what you're doing. (Subject 4)
\end{abstract}

Subject responses regarding the usability of the software were positive, with a median SUS score of 89 (interquartile range of 84-95). Ratings of perceived benefit and desire to use the program were both good, with median scores of 4.0 (interquartile range of 3.0-4.8) and 3.5 (interquartile range of 3.0-4.8) on a 5-point Likert scale, respectively. The preferred timing of program use varied. During a course of home-based intravenous antibiotic treatment or immediately upon hospital discharge was the preferred timing for $70 \%$ of subjects (Table 1).

\section{Secondary Outcomes}

One subject was unable to complete activity monitoring at either time point due to a skin reaction to the SenseWear Pro3 armband. A second subject wore the SenseWear Pro3 armband for insufficient time at the end-intervention mon- itoring period for activity data to be included in the analysis. Four subjects did not complete exercise capacity assessments at the conclusion of the intervention because they either were unable to attend the clinic for assessment $(n=3)$ or were feeling unwell on the day $(n=1)$. Activity monitoring at baseline was undertaken for a mean of $6 \pm 2 \mathrm{~d}$ with an average wear time of $16 \pm 7 \mathrm{~h} / \mathrm{d}$. Moderate-tovigorous physical activity participation was maintained from hospital discharge to end intervention $(P=.2)$. There was no change in time spent sedentary or in mild activity $(P=.8$ and $P=.3$, respectively), and there was no increase in the treatment burden domain of the CFQ-R during the study period $(P=.3)$ (Table 2).

\section{Discussion}

This small study on adults with CF reported feasibility and acceptability of an internet-based program to monitor and encourage physical activity participation. Subjects reported very good usability of the system and perceived the ideal time for use of the program as immediately upon hospital discharge or during the course of home-based intravenous antibiotic treatment. In subjects who completed objective physical activity monitoring, physical activity participation was maintained between hospital discharge and a 2-month follow-up.

Participation in regular exercise and physical activity is encouraged for people with $\mathrm{CF}$, as it can help improve exercise capacity ${ }^{4}$ and is associated with better prognosis. ${ }^{5}$ However, the most effective strategies for encouraging people to undertake and maintain regular physical activity are unclear. The use of self-monitoring via a pedometer in this study was identified as a useful adjunct for tracking activity, although consistency of use may have been limited by the physical appearance of wearing a pedometer. Two uncontrolled cohort studies in children with CF have investigated the use of activity-token rewards and activity counseling with telephone follow-up and pedometer monitoring on exercise participation. ${ }^{30,31}$ Both studies reported an improvement in self-reported activity ${ }^{30}$ or step count ${ }^{31}$; however, adherence to study protocols was poor, and individual variation in activity improvement was large. Although it is unlikely that activity-token rewards would be appropriate for changing exercise behavior in adults, our findings suggest that, if inconspicuous, commercially available activity monitors may be useful for self-monitoring of activity participation.

Technology applications are becoming increasingly common in health-care provision. For people with $\mathrm{CF}$, telehealth technology has been used predominantly for symptom monitoring or transmission of physiologic data and has been found to be both feasible and acceptable. ${ }^{16}$ For people with COPD, telehealth applications have been employed to administer remote and home-based pulmo- 
Table 2. Secondary Outcome Data at Baseline and End Intervention

\begin{tabular}{lcc}
\hline \hline & Baseline & End Intervention \\
\hline Quality of life $(n=10)$ & & $P$ \\
CFQ-R & & $56(41-59)$ \\
$\quad$ Treatment burden & $56(41-56)$ & $44(33-71)$ \\
$\quad$ Respiratory & $33(27-70)$ & $63(29-79)$ \\
$\quad$ Physical & $36(32-67)$ & $6 \pm 1$ \\
Physical activity participation $(n=8)$ & & $15(13-20)$ \\
Activity monitoring (mean \pm SD), & $7 \pm 1$ & $139(103-242)$ \\
Sedentary time, h/d & $15(12-19)$ & $26(9-39)$ \\
Mild activity, min/d & $123(88-177)$ & $.3,447(5,792-9,964)$ \\
Moderate-to-vigorous activity, min/d & $25(13-61)$ & .2 \\
Step count, steps/d & $7,131(6,559-8,369)$ & $640(540-995)$ \\
Exercise capacity $(n=5)$ & $700(570-1,020)$ & .8 \\
Modified Shuttle Test distance, $\mathrm{m}$ & & .3 \\
Values are given as median (interquartile range) unless indicated otherwise. & & .2 \\
CFQ-R = Cystic Fibrosis Questionnaire-Revised & & .2 \\
\hline
\end{tabular}

nary rehabilitation with good effect, ${ }^{32,33}$ and use of a pedometer and internet monitoring of a daily walking program significantly improved monthly step count in men with COPD. ${ }^{14}$ This program also generated graphical feedback of activity for participants, a feature identified as favorable by our subjects. In our study, however, we did not detect an improvement in quantitative measures of physical activity participation, such as daily step count. While quantitative improvement in physical activity participation was not the primary outcome of this study, we may have been less able to identify improvements in parameters such as daily step count as subjects were not prescribed a specific exercise program. Rather, they were encouraged to document participation in their preferred activities. Consequently, when subjects undertook activities such as cycling and swimming, recording step count would not have been possible.

None of the subjects logged into ActivOnline every day, with the average frequency of logins being 4/week. This is consistent with interview comments, in which subjects reported that they preferred to log in every few days or identified forgetting to regularly enter data as a barrier to fulfilling the study requirements. This suggests that, in this group of young people with a chronic disease and high treatment burden, it was the act of regularly reporting their physical activity rather than the frequency of the reporting that was considered useful. The subjects also emphasized the importance of a reminder system, with the current e-mail system or possibly alternative methods such as text messaging, in future modifications of the program. This is in keeping with adults with CF who reviewed a smart phone designed to improve adherence and knowledge for adolescents with CF. ${ }^{34}$ Adults rated most highly reminders and alerts as a useful feature of the CFFONE prototype. ${ }^{34}$ These responses may be a reflection of common reasons for non-adherence with therapeutic regimens by adults with $\mathrm{CF}$ as being too busy or forgetting. ${ }^{8}$

This trial is limited by its small size and recruitment from only a single center. Participation in the study did not increase subject-reported therapy burden on the CFQ-R; however, we did not assess changes in adherence to other therapies as a consequence of trialing ActivOnline.

\section{Conclusions}

This study has shown the use of an internet-based program for monitoring physical activity participation to be feasible and acceptable to adults with CF. Further investigation in the form of a randomized controlled trial is necessary to ascertain the effect of using ActivOnline on improving physical activity participation.

\section{ACKNOWLEDGMENTS}

We thank Stuart Cox and Scott Pederick for their sage advice and enthusiasm for this project.

\section{REFERENCES}

1. World Health Organization. Global recommendations on physical activity and health. 2010. http://www.who.int/dietphysicalactivity/ factsheet_recommendations/en. Accessed October 20, 2014.

2. Kahn EB, Ramsey LT, Brownson RC, Heath GW, Howze EH, Powell KE, et al. The effectiveness of interventions to increase physical activity: a systematic review. Am J Prev Med 2002;22(4 Suppl): 73-107.

3. Australian Bureau of Statistics. Australian health survey: first results 2011-2012. 2012. http://www.abs.gov.au/AUSSTATS/abs@.nsf/Details Page/4364.0.55.0012011-12?OpenDocument. Accessed October 20, 2014. 


\section{Internet-Based Program to Promote Physical Activity in CF}

4. Bradley J, Moran F. Physical training for cystic fibrosis. Cochrane Database Syst Rev 2008;(1):CD002768.

5. Nixon PA, Orenstein DM, Kelsey SF, Doershuk CF. The prognostic value of exercise testing in patients with cystic fibrosis. $\mathrm{N}$ Engl J Med 1992;327(25):1785-1788.

6. Hebestreit H, Kieser S, Rüdiger S, Schenk T, Junge S, Hebestreit A, et al. Physical activity is independently related to aerobic capacity in cystic fibrosis. Eur Respir J 2006;28(4):734-739.

7. Schneiderman-Walker J, Wilkes DL, Strug L, Lands LC, Pollock SL, Selvadurai HC, et al. Sex differences in habitual physical activity and lung function decline in children with cystic fibrosis. J Pediatr 2005;147(3):321-326

8. White D, Stiller K, Haensel N. Adherence of adult cystic fibrosis patients with airway clearance and exercise regimens. J Cyst Fibros 2007;6(3):163-170.

9. Garcia-Aymerich J, Farrero E, Félez MA, Izquierdo J, Marrades RM, Antó JM, Estudi del Factors de Risc d'Agudització de la MPOC Investigators. Risk factors of readmission to hospital for a COPD exacerbation: a prospective study. Thorax 2003;58(2):100-105.

10. García-Polo C, Alcázar-Navarrete B, Ruiz-Iturriaga LA, Herrejón A, Ros-Lucas JA, García-Sidro P, et al. Factors associated with high healthcare resource utilisation among COPD patients. Respir Med 2012;106(12):1734-1742.

11. Garcia-Aymerich J, Lange P, Benet M, Schnohr P, Antó JM. Regular physical activity reduces hospital admission and mortality in chronic obstructive pulmonary disease: a population based cohort study. Thorax 2006;61(9):772-778.

12. Conn VS, Hafdahl AR, Brown SA, Brown LM. Meta-analysis of patient education interventions to increase physical activity among chronically ill adults. Patient Educ Couns 2008;70(2):157-172.

13. Kerem E, Conway S, Elborn S, Heijerman H, Consensus Committee. Standards of care for patients with cystic fibrosis: a European consensus. J Cyst Fibros 2005;4(1):7-26.

14. Moy ML, Weston NA, Wilson EJ, Hess ML, Richardson CR. A pilot study of an Internet walking program and pedometer in COPD. Respir Med 2012;106(9):1342-1350.

15. Nguyen HQ, Gill DP, Wolpin S, Steele BG, Benditt JO. Pilot study of a cell phone-based exercise persistence intervention postrehabilitation for COPD. Int J Chron Obstruct Pulmon Dis 2009;4: 301-313.

16. Cox NS, Alison JA, Rasekaba T, Holland AE. Telehealth in cystic fibrosis: a systematic review. J Telemed Telecare 2012;18(2):72-78.

17. Cox NS, Alison JA, Holland AE. Interventions for promoting physical activity in people with cystic fibrosis. Cochrane Database Syst Rev 2013;(12):CD009448

18. Kosma M, Cardinal BJ, McCubbin JA. A pilot study of a web-based physical activity motivational program for adults with physical disabilities. Disabil Rehabil 2005;27(23):1435-1442.

19. Marcus BH, Ciccolo JT, Sciamanna CN. Using electronic/computer interventions to promote physical activity. Br J Sports Med 2009; 43(2):102-105.
20. Shaw DK. Overview of telehealth and its application to cardiopulmonary physical therapy. Cardiopulm Phys Ther J 2009;20(2):13-18

21. Keating A, Lee AL, Holland AE. Lack of perceived benefit and inadequate transport influence uptake and completion of pulmonary rehabilitation in people with chronic obstructive pulmonary disease: a qualitative study. J Physiother 2011;57(3):183-190.

22. George ES, Kolt GS, Duncan MJ, Caperchione CM, Mummery WK, Vandelanotte $\mathrm{C}$, et al. A review of the effectiveness of physical activity interventions for adult males. Sports Med 2012;42(4): 281-300.

23. Ferney SL, Marshall A. Website physical activity interventions: preferences of potential users. Health Educ Res 2006;21(4):560-566.

24. Britten N. Qualitative interviews in medical research. BMJ 1995; 311(6999):251-253.

25. Brooke J. SUS: a "quick and dirty" usability scale. In: Jordan P, Thomas B, Weerdmeester B, McClelland A. Usability evaluation in industry. London: Taylor and Francis; 1996:189-194.

26. Tullis TS, Stetson JN. A comparison of questionnaires for assessing website usability. http://home.comcast.net/ tomtullis/publications/ UPA2004TullisStetson.pdf. Accessed November 14, 2013.

27. Quittner AL, Buu A, Messer MA, Modi AC, Watrous M. Development and validation of The Cystic Fibrosis Questionnaire in the United States: a health-related quality-of-life measure for cystic fibrosis. Chest 2005;128(4):2347-2354.

28. Bradley J, Howard J, Wallace E, Elborn S. Reliability, repeatability, and sensitivity of the modified shuttle test in adult cystic fibrosis. Chest 2000;117(6):1666-1671.

29. Trost SG, McIver KL, Pate RR. Conducting accelerometer-based activity assessments in field-based research. Med Sci Sports Exerc 2005;37(11):S531-S543.

30. Tuzin B, Mulvihill M, Kilbourn K, Bertran D, Buono MJ, Hovell M, et al. Increasing physical activity of children with cystic fibrosis: a home-based family intervention. Pediatr Exerc Sci 1998;10(1): $57-68$

31. Swisher AK, Moffett K. The effect of coaching on physical activity and quality of life in children and adolescents with cystic fibrosis: a quality improvement pilot study. Internet J Allied Health Sci Pract 2010;8(2):1-7.

32. Stickland M, Jourdain T, Wong EY, Rodgers WM, Jendzjowsky NG, MacDonald GF. Using Telehealth technology to deliver pulmonary rehabilitation to patients with chronic obstructive pulmonary disease. Can Respir J 2011;18(4):216-220.

33. Holland AE, Hill CJ, Rochford P, Fiore J, Berlowitz DJ, McDonald CF. Telerehabilitation for people with chronic obstructive pulmonary disease: feasibility of a simple, real time model of supervised exercise training. J Telemed Telecare 2013;19(4):222-226.

34. Marciel KK, Saiman L, Quittell LM, Dawkins K, Quittner AL. Cell phone intervention to improve adherence: cystic fibrosis care team, patient, and parent perspectives. Pediatr Pulmonol 2010;45(2): 157-164. 